\title{
Distinct Trichoscopic Features of the Sideburns in Frontal Fibrosing Alopecia Compared to the Frontotemporal Scalp
}

\author{
Jessica Cervantes Mariya Miteva
}

Department of Dermatology and Cutaneous Surgery, University of Miami Miller School of Medicine, Miami, FL, USA

\section{Keywords}

Trichoscopy · Alopecia · Frontal fibrosing alopecia .

Sideburns · Hair · Hair disorder · Proximal hair emergence

\begin{abstract}
The trichoscopic features of frontal fibrosing alopecia (FFA) have been described in the frontotemporal area, yet there is no data detailing the trichoscopic features of the sideburns, which can be the initial or exclusive area of involvement. In this retrospective cohort study, 236 trichoscopic images of the frontotemporal and sideburn area obtained via dry trichoscopy from patients with biopsy-proven FFA were examined by two independent researchers to determine the trichoscopic features of FFA in the sideburns. The images of the sideburns were compared to 44 trichoscopic images of 11 healthy volunteers with intact sideburns. Transparent proximal hair emergence surrounded by patches of paler smooth skin was the most prominent finding in the sideburns. Peripilar casts and peripilar erythema were rare in the sideburns compared to the frontotemporal area. Although less common, transparent proximal hair emergence was also seen in the sideburns of healthy controls, yet it was shorter and restricted to individual hairs. Early FFA presenting exclusively or mostly with sideburn involvement should not be
\end{abstract}

\section{KARGER}

(C) 2017 S. Karger AG, Basel

E-Mail karger@karger.com

www.karger.com/sad missed due to absence of peripilar casts and peripilar erythema. Dermoscopy-guided biopsy obtained from hair shafts with transparent proximal hair emergence should be considered to make the diagnosis in this location.

(c) 2017 S. Karger AG, Basel

\section{Introduction}

Frontal fibrosing alopecia (FFA) is a lymphocytic cicatricial alopecia that leads to progressive recession of the frontotemporal hairline most commonly in women. In some cases, hair loss of the eyebrows, eyelashes, trunk, upper and lower extremities [1], moustache and beard area, and sideburns can also occur [2-4]. Exclusive involvement of the sideburns in FFA was reported by us in a single case; however, it may be more prevalent [2]. The trichoscopic findings of FFA have been well described and include loss of follicular openings, peripilar white casts, peripilar erythema, and cicatricial white patches [5]. Peripilar casts, also referred to as follicular hyperkeratosis, represent a concentrically arranged layer or layers of scale attached tightly around the emergence of the hair shafts. In two recent large trichoscopic studies, follicular hyperkeratosis was present in $90 \%$ of the 249

Jessica Cervantes

University of Miami Miller School of Medicine

1475 NW 12th Ave

Miami, FL 33136 (USA)

E-Mail J.Cervantes1@umiami.edu 
Table 1. Summary of the dermographic, clinical and trichoscopic features of 40 patients with FFA

\begin{tabular}{|c|c|}
\hline $\begin{array}{l}\text { Total number of patients with biopsy-proven } \\
\text { diagnosis of FFA and trichoscopic images } \\
\text { stored in the FotoFinder system }\end{array}$ & $n=40$ \\
\hline Gender & Female $(n=38)$, male $(n=2)$ \\
\hline Mean age & 59 years \\
\hline Ethnicity & Caucasian $(n=38)$, Asian $(n=1)$, African American $(n=1)$ \\
\hline Frontotemporal involvement & $38 / 40$ \\
\hline Sideburns involvement & $30 / 40$ \\
\hline Exclusive frontotemporal involvement & $10 / 40$ \\
\hline Exclusive sideburns involvement & $2 / 40$ \\
\hline Trichoscopy of the frontotemporal scalp & $\begin{array}{l}\text { peripilar casts }-24 / 38(63.2 \%) \\
\text { peripilar erythema }-22 / 38(57.9 \%) \\
\text { transparent proximal hair shaft emergence }-6 / 38(15.8 \%) \\
\text { pili torti-like hairs }-6 / 38(15.8 \%) \\
\text { broken hairs - } 4 / 38(10.5 \%)\end{array}$ \\
\hline Trichoscopy of the sideburns region & $\begin{array}{l}\text { transparent proximal hair shaft emergence }-19 / 24^{\mathrm{a}}(79.2 \%) \\
\text { peripilar casts (fine and less adherent) }-4 / 24(16.7 \%) \\
\text { peripilar erythema }-4 / 24(16.7 \%) \\
\text { pili torti-like hairs }-4 / 24(16.7 \%) \\
\text { broken hairs }-2 / 24(8.3 \%)\end{array}$ \\
\hline
\end{tabular}

a 24/30 patients with sideburn involvement had stored trichoscopic images of the area.

patients in the study by Fernandez-Crehuet et al. [6] and in $72.1 \%$ of the 79 patients in the study by Toledo-Pastrana et al. [7], followed by peripilar erythema in 66.3 and $77 \%$, respectively. In the most recently published study on FFA including 242 patients from Spain, perifollicular erythema and hyperkeratosis was found in 114 patients $(47.1 \%)$ at the frontal hairline and in 21 patients $(8.7 \%)$ at the temporal hairline [8]. In our experience, peripilar casts and peripilar erythema are rarely encountered in the sideburn location. Moreover, there is limited literature detailing the trichoscopic features of FFA in this region.

\section{Materials and Methods}

We conducted a retrospective review of the trichoscopic images of patients with biopsy-proven FFA who were seen by one of us (M.M.) at the Department of Dermatology at the University of Miami in the last 2 years. The study was approved by the local IRB. Out of the 108 identified patients, 40 met the criteria of having biopsy-proven diagnosis of FFA and trichoscopic images were obtained via dry trichoscopy (without immersion gel) and stored in

Distinct Trichoscopic Features of the

Sideburns in Frontal Fibrosing Alopecia the FotoFinder computer (Fotofinder Systems, Bad Birnbach, Germany). Only images from baseline visits (average of 3 clinical images per patient, including frontal aspect and both sides and a total of 8 trichoscopic images obtained at $\times 20$ and $\times 40$ magnifications in bilateral frontotemporal scalp and sideburns) were included. Two independent researchers analyzed and compared the trichoscopic images from the frontotemporal scalp and the sideburns for the presence of peripilar erythema, peripilar casts (follicular hyperkeratosis), and transparent hair shaft emergence (visible proximal portions of the hair shafts underneath the skin). The images obtained from the sideburns were compared to 44 trichoscopic images $(\times 20$ and $\times 40)$ of 11 healthy volunteers (10 female and 1 male, mean age 39 years), i.e., clinical staff members who consented to participate. They had no complaint of hair loss, had intact sideburns on clinical examination and declined a history of face-lifting, cosmetic interventions or laser hair removal in this area. The images were obtained and stored via dry trichoscopy (without immersion gel) using the FotoFinder videodermatoscope (Fotofinder Systems).

\section{Results}

The clinical and demographic data are summarized in Table 1. 


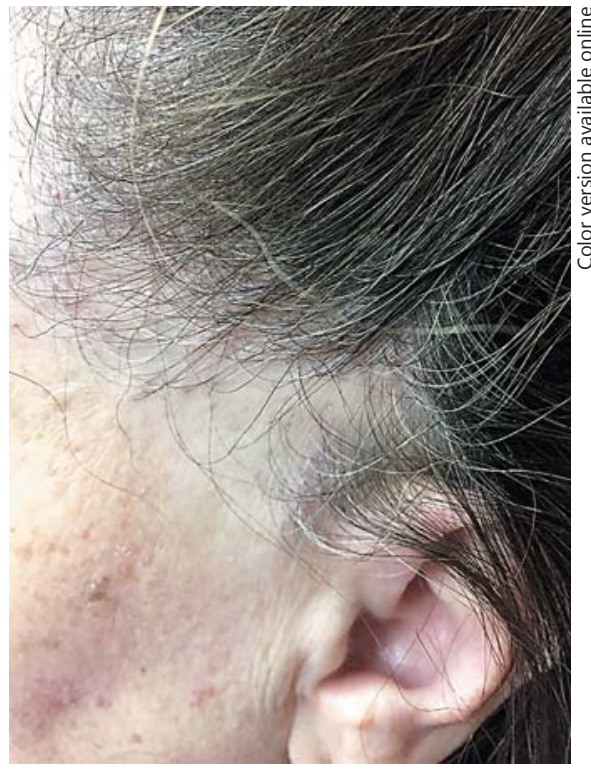

Fig. 1. Sideburn involvement in frontal fibrosing alopecia. Note the pale patches of hairless smooth skin among individual terminal hairs.

\section{Frontotemporal Involvement in FFA}

Our results showed that almost all patients $(38 / 40)$ had involvement of the frontotemporal hairline, of whom 10 had localized pathology to this area. Smooth hairless skin with minimal to no vellus hair was evident on gross examination. Trichoscopy of this area revealed peripilar casts in $63.2 \%(24 / 38)$ and peripilar erythema in 57.9\% (22/38). Transparent hair shaft emergence, i.e., visible proximal portions of the hair shafts underneath the skin, was present in $15.8 \%(6 / 38)$ in this location. The other less common features are summarized in Table 1.

\section{Sideburn Involvement in FFA}

Sideburn involvement was detected in 30 patients (75.0\%), 2 of whom had isolated involvement of these regions without evidence of frontotemporal hairline pathology. In the sideburns, patchy hair loss presenting as hairless, smooth and paler skin with only focally preserved terminal hairs was found in 29 patients (Fig. 1). Complete hair loss of the sideburns was seen in only 1 man. All patients had bilateral involvement. Trichoscopic images of the sideburns were available for 24 patients. Transparent hair shaft emergence, measuring on average $0.55 \mathrm{~mm}$, surrounded by a patch of paler skin was the most common finding in this location $(79.2 \%, 19 / 24)$ (Table 1; Fig. 2a, b). In contrast to the frontotemporal hairline, only 4 of the 24 patients with trichoscopic images of the sideburns showed evidence of peripilar casts in this area, which appeared much finer, more crumbling and less adherent to the shafts (Fig. 2c). Slight peripilar erythema was also observed in 4 out of 24 cases (16.7\%).

\section{Sideburns in Healthy Controls}

The sideburns of the healthy controls showed preserved follicular density with preserved vellus hairs. No peripilar erythema was detected. In 1 case, there was fine crumbling and a less adherent peripilar cast around 2 hairs. Transparent proximal hair emergence was visible in $5 / 11$ cases $(45.4 \%)$, all of which were restricted to individual hairs and measured on average $0.19 \mathrm{~mm}$ (Fig. 3).

\section{Discussion}

Our study demonstrates that the trichoscopic features of the sideburn region derail from the typical trichoscopic features of FFA, while the trichoscopic findings of the frontotemporal scalp are similar to previous studies $[6,7]$. In the current study, transparent proximal hair emergence was the most prominent finding in the sideburns (79.2\%), and was not accompanied by peripilar casts, further supporting the noninflammatory pattern of FFA in this location. Others have reported that FFA can be noninflammatory on clinical presentation in locations such as the sideburns, eyebrows, trunk, and limbs [9]. We have observed that in FFA of the limbs, trichoscopy does not reveal peripilar erythema or peripilar casts, but often shows transparent proximal hair emergence (authors' unpublished data).

Transparent proximal hair emergence in the sideburns does not appear to be specific for FFA as 5/11 controls revealed similar findings. However, there are a few differences: (1) the average length of the transparent proximal hair emergence in FFA was $0.55 \mathrm{~mm}$ versus only $0.19 \mathrm{~mm}$ in healthy controls; (2) only 1 or 2 hair shafts showed that feature in healthy controls versus most of the hair shafts in the affected sideburns in FFA; and (3) a patch of paler skin surrounded the transparent proximal emergence in FFA versus normal skin in healthy sideburns.

The explanation for the higher prevalence of proximal hair emergence in the sideburn location compared to the frontotemporal scalp in FFA (79.2 vs. 15.8\%) is most likely complex. A topographic study of the head has shown that the skin in the sideburn area is normally thinner than the skin on the frontal scalp [10]. Skin atrophy of the sideburns, observed in $54.5 \%$ of our cases as patches of pale 

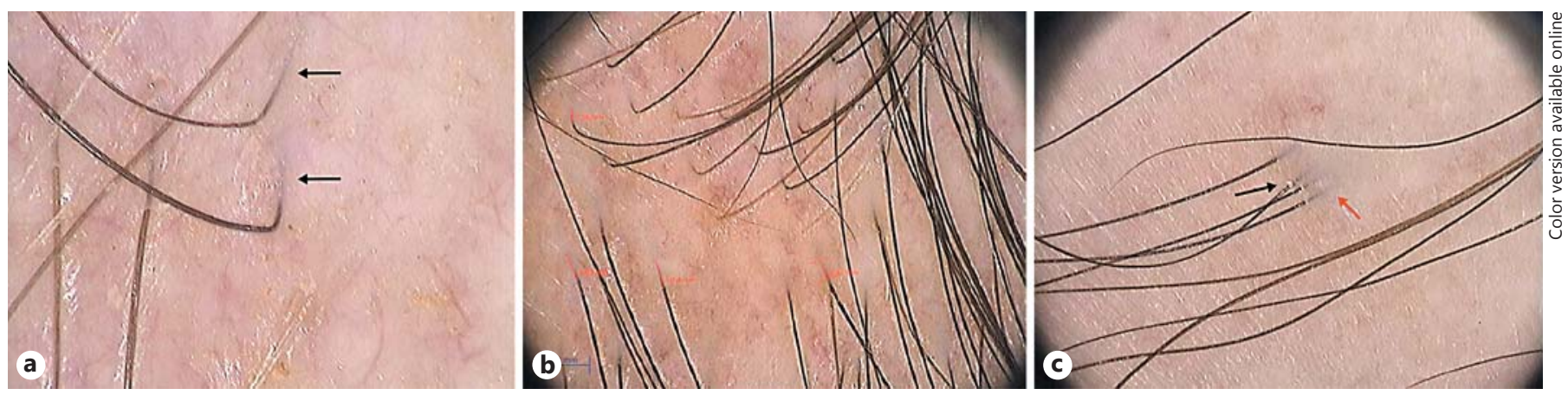

Fig. 2. a Trichoscopy of the sideburns in frontal fibrosing alopecia is characterized by loss of follicular openings and transparent proximal hair shaft emergence, which are segments of the proximal hair shafts that are visible underneath the skin (arrows). Note the patches of pale atrophic skin around them (FotoFinder Systems; $\times 50$ ). $\mathbf{b}$ Trichoscopy of the sideburns from a different case of frontal fibrosing alopecia shows similar features. Note the absence of vellus hairs and the presence of transparent proximal hair shaft emergence of an average length of $0.53 \mathrm{~mm}$ in this case (FotoFinder Systems; $\times 40$ ). c Trichoscopy of the sideburns from a third case of frontal fibrosing alopecia shows loss of follicular openings and 4 transparent proximal hair shaft emergences surrounded by a patch of pale smooth skin (red arrow). There is a very fine, less adherent hair cast around two emergences (black arrow) (FotoFinder Systems; $\times 40$ ).

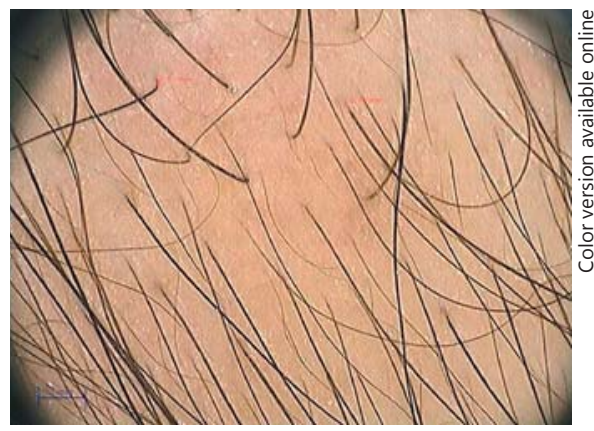

Fig. 3. Trichoscopy of the sideburns of healthy controls. Note the preserved follicular density with preserved vellus hairs and absence of peripilar erythema. Two hair shafts show transparent proximal hair shaft emergence with an average length of $0.16 \mathrm{~mm}$ (FotoFinder Systems; ×40).

white, thin and smooth skin, seen in conjunction with transparent proximal hair shaft emergence or in an interfollicular pattern may be another contributing factor. However, at this point, it is unclear if a correlation between this trichoscopic feature and the pathology exists due to the small number of biopsies from the sideburns. Dermoscopy-guided, 3-mm punch biopsies from sideburn involvement were available for 3 cases and in addition to the classic features of FFA, the presence of skeletal muscle was found in 1 case confirming the degree of skin atrophy in this location.

Distinct Trichoscopic Features of the Sideburns in Frontal Fibrosing Alopecia
Skin atrophy might not be the only cause for the translucent proximal hair shafts in FFA of the sideburns. In our experience, based also on reviewing biopsies from limb involvement in FFA, the absence of fibrosis may also contribute to this finding. Another possible factor that may favor visualization of proximal hair shafts in the sideburns is the direction of the hair shaft; that is, while in the frontotemporal scalp they may be more perpendicularly implanted, in the sideburns they seem to lean and exit the skin in a more inclined fashion, allowing visualization of their "path" underneath the skin.

In conclusion, early FFA presenting exclusively or mostly with sideburn involvement should not be misdiagnosed as traction alopecia or alopecia areata due to absence of perpilar casts and peripilar erythema. In alopecia areata, patches are usually asymmetric and ophiasis has a characteristic clinical presentation. There are trichoscopic findings of yellow dots, black dots and broken hairs, including exclamation hairs. In traction alopecia, the vellus hairs are usually preserved, which is designated as the fringe sign. In cases of active traction, some hairs at the margin of the patches show cylindrical structures that envelop the proximal portions of the hair casts $[5,11]$.

While dermoscopy-guided biopsy of hair shafts with peripilar erythema and casts is usually diagnostic for FFA and lichen planopilaris [12], in FFA of the sideburns the transparent proximal hair shaft emergence, rather than the surrounding smooth hairless skin, can provide a good source for dermoscopy-guided biopsy.

Skin Appendage Disord 2018;4:50-54 DOI: $10.1159 / 000479116$ 
The main limitation of our study was that the majority of patients were Caucasian (38/40). The greater presence of pigments in skin of patients with darker skin types may hamper visualization of the features reported in this study.

\section{Acknowledgement}

We are indebted to the patients for granting permission to publish this information.

\section{Statement of Ethics}

Verbal photographic and informed consent was obtained from the patients described in this article. This study was approved by the University of Miami Institutional Review Board and was conducted in accordance with the ethical standards of the Human Subject Research Office at the University of Miami.

\section{Disclosure Statement}

The authors have no conflict of interest to declare. There were no funding sources for this work.

\section{References}

1 Miteva M, Camacho I, Romanelli P, Tosti A: Acute hair loss on the limbs in frontal fibrosing alopecia: a clinicopathological study of two cases. Br J Dermatol 2010;163:426-428.

2 AlGaadi S, Miteva M, Tosti A: Frontal fibrosing alopecia in a male presenting with sideburn loss. Int J Trichology 2015;7:72-73.

3 Vano-Galvan S, Molina-Ruiz AM, SerranoFalcon C, Arias-Santiago S, Rodrigues-Barata AR, Garnacho-Saucedo G, Martorell-Calatayud A, Fernandez-Crehuet P, Grimalt R, Aranegui B, Grillo E, Diaz-Ley B, Salido R, Perez-Gala S, Serrano S, Moreno JC, Jaen P, Camacho FM: Frontal fibrosing alopecia: a multicenter review of 355 patients. J Am Acad Dermatol 2014;70:670-678.

4 Ormaechea-Perez N, Lopez-Pestana A, Zubizarreta-Salvador J, Jaka-Moreno A, PanesRodriguez A, Tuneu-Valls A: Frontal fibrosing alopecia in men: presentations in 12 cases and a review of the literature. Actas Dermosifiliogr 2016;107:836-844.
5 Miteva M, Tosti A: Hair and scalp dermatoscopy. J Am Acad Dermatol 2012;67:10401048.

6 Fernandez-Crehuet P, Rodrigues-Barata AR, Vano-Galvan S, Serrano-Falcon C, MolinaRuiz AM, Arias-Santiago S, Martorell-Calatayud A, Grimalt R, Garnacho-Saucedo G, Serrano S, Carlos Moreno J, Jaen P, CamachoMartinez FM: Trichoscopic features of frontal fibrosing alopecia: results in 249 patients. J Am Acad Dermatol 2015;72:357-359.

7 Toledo-Pastrana T, Hernandez MJ, Camacho Martinez FM: Perifollicular erythema as a trichoscopy sign of progression in frontal fibrosing alopecia. Int J Trichology 2013;5: 151-153.
8 Moreno-Arrones OM, Saceda-Corralo D, Fonda-Pascual P, Rodrigues-Barata AR, Buendia-Castano D, Alegre-Sanchez A, Pindado-Ortega C, Molins M, Perosanz D, Segurado-Miravalles G, Jaen P, Vano-Galvan S: Frontal fibrosing alopecia: clinical and prognostic classification. J Eur Acad Dermatol Venereol 2017, Epub ahead of print.

9 Ramaswamy P, Mendese G, Goldberg LJ: Scarring alopecia of the sideburns: a unique presentation of frontal fibrosing alopecia in men. Arch Dermatol 2012;148:1095-1096.

10 Chopra K, Calva D, Sosin M, Tadisina KK, Banda A, De La Cruz C, Chaudhry MR, Legesse T, Drachenberg CB, Manson PN, Christy MR: A comprehensive examination of topographic thickness of skin in the human face. Aesthet Surg J 2015;35:1007-1013.

11 Tosti A, Tosti A: Dermoscopy of the Hair and Nails, ed 2. Boca Raton, CRC Press, 2016.

12 Miteva M, Tosti A: Dermoscopy guided scalp biopsy in cicatricial alopecia. J Eur Acad Dermatol Venereol 2013;27:1299-1303. 